\title{
SPECTROSCOPY
}

\section{Diamonds are a spectroscopist's best friend}

A diamond impurity holds great promise for nanoscale magneto-optical resonance imaging.

A common, yellowish diamond impurity called a nitrogen-vacancy center decreases diamonds' value as jewels, but the groups of Fedor Jelezko of the Universität Stuttgart and Mikhail Lukin of Harvard University recently showed that this impurity has exceptional value for novel spectroscopic applications.

The strong, stable fluorescence of nitrogenvacancy centers and nontoxicity of diamond are useful for optical imaging. The impurity also has an unpaired electronic spin, enabling magnetic sensing. "The fluorescence of this center depends on magnetic fields, so information about the magnetic field can be encoded in fluorescence or vice versa," explains Jelezko. His group imaged single nitrogen-vacancy centers in diamond nanocrystals with an atomic force microscope
(AFM) combined with a confocal microscope (Balasubramanian et al., 2008). Incorporating this nanocrystal into an AFM tip, they also used it as a scanning probe to map magnetic fields with nanometer resolution. "Ideally, you could do magnetic resonance imaging with atomic resolution," says Jelezko.

Magnetic resonance techniques work by detecting the response of nuclear or electronic spins to an applied magnetic field, but these methods are notoriously insensitive. "A holy grail in the field is to extend such techniques down to the level of single molecules," says Lukin. To do so, he explains, "one has to have a very good and very small magnetic sensor." His group showed that a single nitrogen impurity in a diamond nanocrystal serves as such a sensor (Maze et al., 2008). Because it is so small and sensitive, it can be brought into direct proximity with single nuclear or electronic spins, potentially enabling their detection. The sensor works at room temperature, so applications in living cells should be possible.

What is truly exciting about these two papers is that "if one would combine these two advances, then you would have a scanning magnetic sensor with an extraordinary combination of sensitivity and spatial resolution," notes Lukin. If the technique can be extended to detect single nuclear spins, "that would open new doors [for resolving] the three-dimensional structure of a single protein," says Jelezko. "There is a lot of work to do, but I think it's possible."

\section{Allison Doerr}

\section{RESEARCH PAPERS}

Balasubramanian, G. et al. Nanoscale imaging magnetometry with diamond spins under ambient conditions. Nature 455, 648-651 (2008).

Maze, J.R. et al. Nanoscale magnetic sensing with an individual electronic spin in diamond. Nature $\mathbf{4 5 5}$, 644-647 (2008). 